\title{
Droplet digital PCR using HER2/EIF2C1 ratio for detection of HER2 amplification in breast cancer tissues
}

\author{
Anchalee Tantiwetrueangdet ${ }^{1} \cdot$ Ravat Panvichian $^{2}\left(\mathbb{D} \cdot\right.$ Sansanee Wongwaisayawan $^{3} \cdot$ Natthaporn Sueangoen $^{1}$. \\ Panuwat Lertsithichai ${ }^{4}$
}

Received: 5 September 2018 / Accepted: 26 September 2018 / Published online: 3 October 2018

(c) The Author(s) 2018

\begin{abstract}
Breast cancers with amplification and overexpression of human epithelial growth factor receptor 2 (HER2) are associated with poor prognosis, and targeted for anti-HER2 therapy. Immunohistochemistry (IHC) and fluorescence in situ hybridization (FISH) are currently the recommended methods to asses HER2 overexpression/amplification. Droplet digital PCR (ddPCR), a highly accurate method to quantify DNA copy number, is potentially a robust alternative for HER2 diagnostics. In the FISH assay and most of previous ddPCR reports, chromosome 17 centromere (CEP17) has been used as the reference control to determine HER2/CEP17 ratio. Nevertheless, miss-classification could occur when HER2 is co-amplified with CEP17. To avoid this inherent defect, in the present study, we employed ddPCR assay using the human eukaryotic translation initiation factor 2C1 (EIF2C1) gene located at chromosome 1p34.3 as the reference control to quantify HER2 copy number in 31 frozen breast cancer tissues. HER2 status of these samples had been determined by FISH and classified as HER2-amplified and HER2-non-amplified breast cancers. The results showed that HER2 determined by ddPCR using HER2/EIF2C1 ratio was in good concordance with HER2 determined by FISH using HER2/CEP17 ratio, the concordance rate 87.1\% (27/31), Kappa $=0.719$. The sensitivity and specificity of ddPCR assay was $90 \%(9 / 10)$ and $85.7 \%(18 / 21)$, respectively. The median HER2/EIF2C1 copy number ratio in HER2-amplified cancers (6.55, range 1.3-17.3) was significantly higher than in HER2non-amplified cancers $(1.05$, range $0.6-3.6, p<0.001)$. This study demonstrated that ddPCR using HER2/EIF2C1 ratio could accurately assess HER 2 status in frozen breast cancer tissues. Thus, our findings warrant further studies into breast cancer with HER2-equivocal by IHC/FISH.
\end{abstract}

Keywords ddPCR $\cdot$ EIF2C $1 \cdot$ FISH $\cdot$ HER2 $\cdot$ IHC

\section{Introduction}

Breast cancer is a heterogeneous disease and can be categorized into different subtypes based on expression of estrogen receptor (ER), progesterone receptor (PR), and human epidermal growth factor receptor-2 (HER2), which

Ravat Panvichian

ravat.pan@mahidol.ac.th

Anchalee Tantiwetrueangdet

anchalee.rat@mahidol.ac.th

Sansanee Wongwaisayawan

sansanee.won@mahidol.ac.th

Natthaporn Sueangoen

natthaporn.sue@mahidol.ac.th

Panuwat Lertsithichai

panuwat.ler@mahidol.ac.th have different prognosis and treatment [1]. Amplification and overexpression of HER2/Neu are detected in approximately $25-30 \%$ of breast cancers and are strongly associated with poor prognosis [2, 3]. In addition, HER2 status has a therapeutic impact because monoclonal antibodies against HER2 (Trastuzumab, Pertuzumab, Trastuzumab

1 Research Center, Faculty of Medicine, Ramathibodi Hospital, Mahidol University, Bangkok, Thailand

2 Department of Internal Medicine, Division of Medical Oncology, Faculty of Medicine, Ramathibodi Hospital, Mahidol University, Rama 6 Road, Rajthevi, Bangkok 10400, Thailand

3 Department of Pathology, Faculty of Medicine, Ramathibodi Hospital, Mahidol University, Bangkok, Thailand

4 Department of Surgery, Faculty of Medicine, Ramathibodi Hospital, Mahidol University, Bangkok, Thailand 
emtansine) have been shown to be effective for treating HER2-positive breast cancer [4-9].

Immunohistochemistry (IHC) and fluorescence in situ hybridization (FISH) are currently the methods recommended by ASCO/CAP guidelines to asses HER2 status $[10,11]$. Most laboratories initially investigate HER 2 status with IHC which is easier to perform, but analysis of the results could be subjective and varied with different antibodies and observers. In contrast to IHC, FISH technique offers better diagnostic accuracy and added confidence, particularly when it is used to supplement weak IHC signals, but it is more labor intensive, time-consuming, and expensive.

Recently, droplet digital PCR (ddPCR) has been developed for absolute nucleic acid quantification [12]. Droplet digital PCR (ddPCR) is potentially an alternative technique to achieve a higher throughput capability and may yield a more accurate diagnosis for HER2 amplification. Concordance between HER2 status from ddPCR assays and HER2 status from standard HER2 assays has been reported [13-18]. In standard FISH assay, chromosome 17 centromere (CEP17) has been used as the reference control to determine HER2/CEP17 copy number ratio. However, misclassification of patients as HER2 non-amplified due to focal amplification of chromosome 17 centromere (CEP17) which leads to amplification of the reference region has been reported [19]. An alternative reference FISH probes have been suggested such as SMS, RARA, and TP53 genes to determine the true HER2 amplification status in patients with polysomy of chromosome 17 [19]. Nevertheless, most of the previous studies on detection of HER 2 amplification by ddPCR still used CEP17 region as the reference control $[13,15-18]$. Only one study used elongation factor Tu GTP binding domain containing 2 (EFTUD2) gene located at $17 \mathrm{q} 21.31$ as an alternative chromosome 17 probe [14].

It might be worthy to seek for other reference probes that can identify true HER2 amplification, especially in CEP17 co-amplification cases. The human eukaryotic translation initiation factor 2C1 (EIF2C1) gene located at $1 \mathrm{p} 34.3$ has been suggested by Bio-Rad as a reference control for HER2 copy number quantification. However, HER 2 status measurement in breast cancer determined by ddPCR using EIF2C1 as the reference control has not been reported before. Thus, in this study, we sought to quantify HER 2 amplification in breast cancer tissues by employing ddPCR assay using EIF2C as the reference control and compared our results with those obtained by FISH assay for the same samples. HER2 status determined by FISH was considered according to the 2013 ASCO/CAP HER2 guideline, and HER2/CEP17 ratio $\geq 2$ was considered as HER2 amplification [11].

\section{Materials and methods}

\section{DNA extraction}

Breast cancer tissues have been obtained from Ramathibodi Hospital and kept at $-80{ }^{\circ} \mathrm{C}$. HER2 status of these samples had been determined by FISH/or IHC in our previous report [20]. The study protocol was approved by the Ethics Committee of the Faculty of Medicine, Ramathibodi Hospital, Mahidol University.

DNA of 31 samples snap frozen breast cancer tissues which have been confirmed by an experienced breast pathologist were isolated with QIAamp DNA Tissue Kit (Qiagen, Valencia, CA, USA) according to the manufacturer's instructions and the quantity was measured by Nanodrop.

\section{Digital PCR}

Digital PCR was performed using the droplet digital PCR (ddPCR) method on Bio-Rad QX200 ${ }^{\mathrm{TM}}$ (Bio-Rad, Hercules, CA, USA). A total $20 \mu \mathrm{l}$ PCR reaction was prepared with 15-20 ng DNA and 2X ddPCR Supermix for probe (BioRad, Hercules, CA, USA); primers and fluorescent probes (FAM and VIC) were prepared from Prime PCR assay for ddPCR (dHsaCP1000116 for HER2 and dHsaCP2500349 for EIF2C1 as the reference control). HindIII was mixed in PCR reaction. Droplets were generated by Bio-Rad QX200 droplet generator. Then, the total $40 \mu \mathrm{l}$ of emulsified PCR reactions were transferred to a 96-well plate and heat sealed before running on T1000 thermal cycler (Bio-Rad, Hercules, CA, USA) with the following cycle: $95^{\circ} \mathrm{C}$ for $10 \mathrm{~min}, 40$ cycles of $94^{\circ} \mathrm{C}$ for $30 \mathrm{~s}$ and $60^{\circ} \mathrm{C}$ for $60 \mathrm{~s}, 98^{\circ} \mathrm{C}$ for $10 \mathrm{~min}$, and hold at $4{ }^{\circ} \mathrm{C}$. The temperature ramp rate was $2^{\circ} \mathrm{C} / \mathrm{s}$ for all steps. Negative control with no DNA was included in each run. After the PCR, the PCR plates were transferred to Bio-Rad QX200 droplet reader. Analysis of ddPCR data was performed by using QuantaSoft v1.3.2.0 software from Bio-Rad. HER2/EIF2C1 ratio $\geq 2.0$ was defined as HER2 amplification, and HER2/EIF2C1 ratio $<2.0$ was defined as HER2 non-amplification.

\section{Fluorescence in situ hybridization (FISH)}

FISH analysis was performed in isolated nuclei using the PathVysion HER2 DNA probe kit (Vysis, Illinois, USA) following the manufacturer's protocol as previously published [20]. As proposed by the ASCO/CAP guideline [11], HER2/ CEP17 ratio $<2$ was considered HER2 negative, and HER2/ CEP17 ratio $\geq 2$ was considered HER2 positive. 


\section{Immunohistochemistry (IHC)}

IHC for HER2 overexpression was performed according to standard methods as previously described [20] and scored according to the ASCO/CAP guideline as negative $(0,1+)$, equivocal $(2+)$, or positive $(3+)[11]$.

\section{Statistical analysis}

Statistical analyses were performed with SPSS v.11.5 (SPSS Inc., Chicago, Illinois, USA) or GraphPad Prism 7 (version 7.03). Kappa coefficient was used to determine the concordance between FISH and ddPCR method in detecting HER2 amplification.

\section{Results}

In this study, HER2 amplification was determined in 31 frozen breast cancer tissues by ddPCR. HER 2 status of these samples had been determined by FISH/or IHC in the previous report [20]. Of the 31 breast cancer tissues, HER2 status was defined by FISH as HER2-amplified and HER2-nonamplified breast cancers. Clinicopathologic characteristics of patients with invasive breast carcinomas are shown in Table 1. We assessed the potential of ddPCR using HER2/ EIF2C1 copy number ratio for detection of HER2 amplification. HER2/EIF2C1 copy number ratio in each sample was determined in three different experiments. Representative of ddPCR plots are shown in Fig. 1. Data of HER2 status determined by IHC, FISH, and ddPCR are shown in Table 2 . The reproducibility of three replicates was tight

Table 1 Clinicopathologic characteristics of patients with invasive breast carcinomas $(n=31)$

\begin{tabular}{lll}
\hline Characteristics & Number of patients & $\%$ \\
\hline Age (years) & & \\
$\quad<50$ & 16 & 51.6 \\
$>50$ & 15 & 48.4 \\
Tumor grade & & 58 \\
$1-2$ & 18 & 42 \\
3 & 13 & \\
Lymph node status & & 38.7 \\
Negative & 12 & 61.3 \\
Positive & 19 & \\
Estrogen receptor status & & 64.5 \\
$\quad$ Negative & 20 & 35.5 \\
Positive & 11 & 64.5 \\
HER2 status & & 35.5 \\
$\quad$ Negative & 21 & \\
Positive & 10 & \\
\hline
\end{tabular}

as shown in Fig. 2. The threshold for the HER2/EIF2C1 copy number ratio $\geq 2.0$ was defined as HER2 amplification, consistently with ASCO/CAP guideline for HER2/CEP17 ratio [11]. The median HER2/EIF2C1copy number ratio in HER2-amplified breast cancers (6.55, range 1.3-17.3) was significantly higher than in HER2-non-amplified breast cancers (1.05, range 0.6-3.6, $p=0.000$ Mann-Whitney $U$ test), as shown in Fig. 3. HER2 status determined by ddPCR using HER2/EIF2C1 ratio was in good concordance with HER2 status determined by FISH using HER2/CEP17 ratio, the concordance rate $87.1 \%(27 / 31)$, Kappa $=0.719$. The sensitivity and specificity of ddPCR assay was $90 \%$ (9/10) and $85.7 \%$ (18/21), respectively, as shown in Table 3.

\section{Discussion}

Due to the prognostic and therapeutic impacts, correct identification of patients with HER2 amplification is crucial. There are several methods to determine HER2 status. However, FISH and IHC are currently the methods recommended by ASCO/CAP guidelines [10, 11]. Most laboratories initially investigate HER2 status with IHC and the result scored as $1+$ (negative), $2+$ (equivocal), and $3+$ (positive). IHC analysis is prone to the subjective evaluation of images and the results can be varied with different antibodies and observers. FISH analysis usually uses a probe to the HER2 gene and another for CEP17, as the reference control. An HER2/CEP17 ratio of less than 1.8 is considered negative, $1.8-2.0$ is considered equivocal, and more than 2.0 is considered positive for HER2 amplification, as the ASCO/CAP guidelines [11]. FISH is more labor intensive, time-consuming, and expensive method. In addition, several studies [19, 21-23] have reported that true polysomy of chromosome 17 is rare. Patients with increased HER2 copy numbers along with increased CEP17 copy numbers might be misclassified as non-amplified HER2. In principle, ddPCR and FISH methods are both based on nucleic acid level. Absolute HER2 copy number can be determined by ddPCR without the need for calibration curves. This technique may be useful as an alternative to IHC/FISH. The advantage of this method is that it enables objective evaluation, robustness, and high throughput.

In this study, the concordance rate for HER2 detection by ddPCR and FISH was $87.1 \%$ (27/31), with 3 samples (B16, B24, and B48) that were classified as HER2 amplified by ddPCR but HER 2 non-amplified by FISH, as shown in Tables 2 and 3. There was only one sample (B37) which was classified as HER2 non-amplified (ratio = 1.3) by ddPCR but HER2 amplified (uncountable) by FISH. CEP17 did not gain in both B16 and B48, and IHC showed $2+$ in B16; therefore, the discordance in these samples might not be due to CEP17 gain. For B24, CEP17 gained, IHC showed 2+, 
A High level HER2 amplification, HER2/EIF2C1 ratio=14 (case B43)

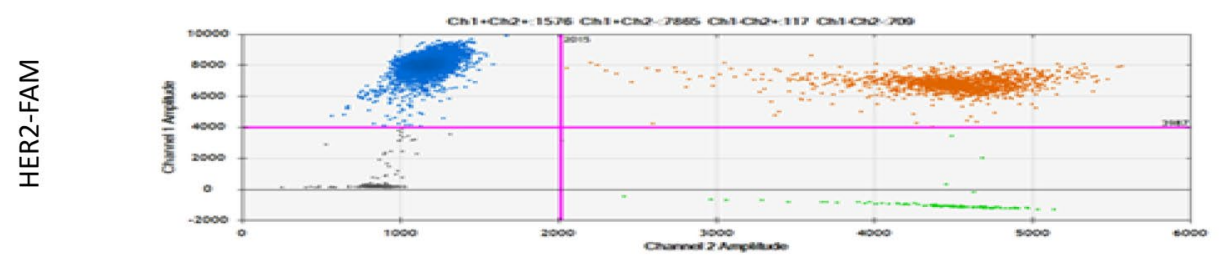

EIF2C1-VIC

B Low level HER2 amplification, HER2/EIF2C ratio=3.6 (case B16)

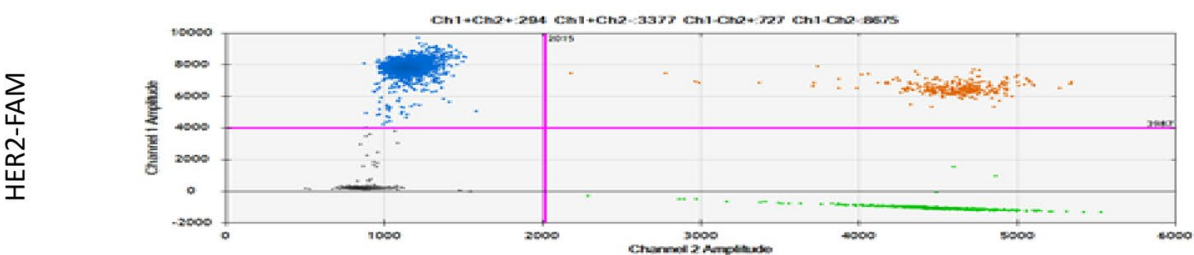

EIF2C1-VIC

C HER2 non-amplification, HER2/EIF2C1 ratio=0.9 (case B7)

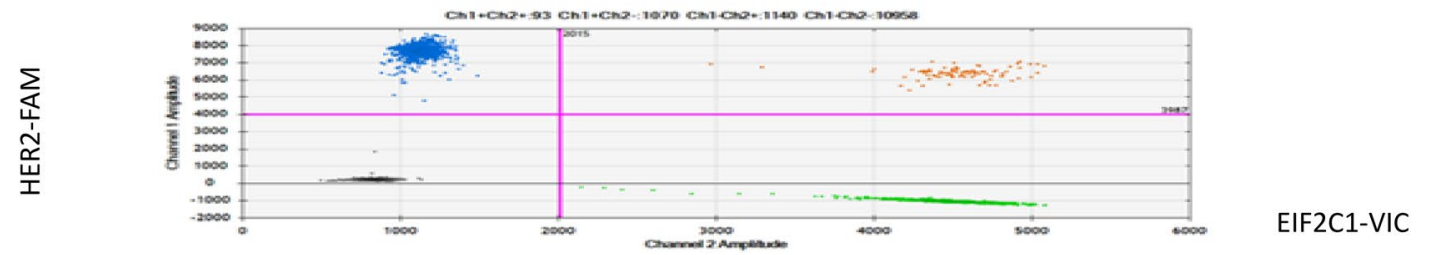

Fig. 1 Representative of HER2 amplification detected by ddPCR in breast cancer tissues using EIF2C1 as the reference control. HER2 and EIF2C1 were labeled with FAM and VIC fluorescent probe, respectively. a High-level HER2 amplification, b low-level HER2

and ddPCR showed low-level amplification (ratio=2.1). The discordance of HER2 status in B37 sample might be due to intratumoral heterogeneity of the sample. Copy number analysis by ddPCR is based on an average of all cell DNA in the sample. Therefore, to maintain sensitivity in the sample which is contaminated with high normal DNA, the lower ratio should be applied [14]. Ten samples with high-level HER2 amplified by FISH showed HER2 copy amplification as a cluster, suggesting that the amplification of these samples was homogeneous staining region type (HSR). Of these samples, FISH could not detect accurate copy number due to uncountable positive signals while accurate copy number could be achieved by ddPCR. Of the 10 samples with highlevel HER2 amplification by FISH, CEP17 gain occurred in amplification, c HER2 non-amplification. In each subfigure, the four quadrants represent top left: droplets with HER2 DNA only, top right: droplets with both HER2 and EIF2C1 DNA, bottom right: droplets with EIF2C1 DNA only, and bottom left: droplets with no DNA

6 samples. From these results, we observed that CEP17 gain did not affect HER2 classification in the samples with highlevel HER2 amplification by FISH. The good concordance between HER2 amplification by ddPCR and HER2 amplification by FISH in breast cancer has been reported [13-17]. Among these prior studies, most of them used CEP17 region as the reference control [13, 15-17]; only one study used EFTUD2 as an alternative chromosome 17 probes [14].

This study demonstrated that ddPCR using HER2/ EIF2C1 ratio could accurately assess HER2 status in frozen breast cancer tissues. Thus, our findings warrant further studies to examine whether ddPCR using HER2/EIF2C1 ratio could discriminate the breast cancer with HER2-equivocal by IHC/FISH. 
Table 2 HER 2 status determined by IHC, FISH, and ddPCR

\begin{tabular}{|c|c|c|c|c|c|c|}
\hline \multirow{2}{*}{$\begin{array}{l}\text { Case no. } \\
\text { B1 }\end{array}$} & \multirow{2}{*}{$\begin{array}{l}\text { IHC } \\
\text { ND }\end{array}$} & \multirow{2}{*}{$\begin{array}{l}\text { HER2/CEP17 ratio } \\
\text { (FISH) } \\
\text { Amp }\end{array}$} & \multicolumn{3}{|c|}{$\begin{array}{l}\text { HER2/EIF2C1 ratio } \\
\text { Triplicate (ddPCR) }\end{array}$} & \multirow{2}{*}{$\begin{array}{c}\text { HER2/EIF2C1 ratio } \\
\text { Mean } \pm \text { SD (ddPCR) } \\
8.8 \pm 0.20\end{array}$} \\
\hline & & & 9.0 & 8.6 & 8.8 & \\
\hline B2 & ND & 1.0 & 1.0 & 1.0 & 0.9 & $1.0 \pm 0.06$ \\
\hline B4 & $3+$ & Amp & 8.4 & 7.9 & 7.7 & $8.0 \pm 0.36$ \\
\hline B5 & $3+$ & 0.8 & 0.9 & 0.9 & 1.2 & $1.0 \pm 0.17$ \\
\hline B7 & ND & 1.1 & 0.9 & 0.9 & 0.9 & $0.9 \pm 0.00$ \\
\hline B8 & $1+$ & 1.1 & 1.0 & 1.0 & 0.9 & $1.0 \pm 0.06$ \\
\hline B9 & $\mathrm{ND}$ & 1.8 & 0.6 & 0.6 & 0.7 & $0.6 \pm 0.06$ \\
\hline B11 & $3+$ & Amp & 6.1 & 6.0 & 5.6 & $5.9 \pm 0.26$ \\
\hline B13 & $1+$ & 1.1 & 1.3 & 1.3 & 1.2 & $1.3 \pm 0.06$ \\
\hline B14 & $3+$ & Amp & 4.2 & 4.1 & 4.2 & $4.2 \pm 0.06$ \\
\hline B15 & $2+$ & 1.1 & 1.2 & 1.2 & 1.0 & $1.1 \pm 0.12$ \\
\hline B16 & $2+$ & 1.1 & 3.6 & 3.6 & 3.6 & $3.6 \pm 0.00$ \\
\hline B18 & $1+$ & 1.0 & 1.1 & 1.1 & 1.1 & $1.1 \pm 0.00$ \\
\hline B19 & $3+$ & Amp & 18.5 & 16.3 & 17.2 & $17.3 \pm 1.11$ \\
\hline B21 & 0 & 1.9 & 1.4 & 1.3 & 1.4 & $1.4 \pm 0.06$ \\
\hline B23 & $1+$ & 1.0 & 1.0 & 1.0 & 1.1 & $1.0 \pm 0.06$ \\
\hline B24 & $2+$ & 1.0 & 1.9 & 2.0 & 2.3 & $2.1 \pm 0.21$ \\
\hline B25 & ND & 1.7 & 1.1 & 1.2 & 1.2 & $1.2 \pm 0.06$ \\
\hline B26 & ND & 1.5 & 1.3 & 1.3 & 1.5 & $1.4 \pm 0.12$ \\
\hline B28 & $3+$ & Amp & 6.9 & 6.6 & 6.9 & $6.8 \pm 0.17$ \\
\hline B29 & $\mathrm{ND}$ & Amp & 6.3 & 6.5 & 6.2 & $6.3 \pm 0.15$ \\
\hline B32 & ND & Amp & 2.7 & 2.5 & 2.6 & $2.6 \pm 0.10$ \\
\hline B35 & ND & 1.1 & 1.0 & 0.9 & 0.9 & $0.9 \pm 0.06$ \\
\hline B36 & $\mathrm{ND}$ & 1.1 & 1.0 & 1.0 & 1.0 & $1.0 \pm 0.00$ \\
\hline B37 & $\mathrm{ND}$ & Amp & 1.3 & 1.3 & 1.3 & $1.3 \pm 0.00$ \\
\hline B38 & $\mathrm{ND}$ & 1.6 & 1.1 & 1.05 & 1.1 & $1.1 \pm 0.30$ \\
\hline B40 & $\mathrm{ND}$ & 1.0 & 0.7 & 0.7 & 0.7 & $0.7 \pm 0.00$ \\
\hline B41 & ND & 1.0 & 1.3 & 1.2 & 1.2 & $1.2 \pm 0.06$ \\
\hline B43 & $3+$ & Amp & 14.1 & 13.8 & 14.2 & $14.0 \pm 0.21$ \\
\hline B48 & $\mathrm{ND}$ & 1.0 & 6.7 & 7.2 & 7.0 & $7.0 \pm 0.25$ \\
\hline B62 & $1+$ & 1.0 & 1.1 & 1.1 & 1.1 & $1.1 \pm 0.00$ \\
\hline
\end{tabular}

Amp HER2 amplification as cluster, $N D$ not done

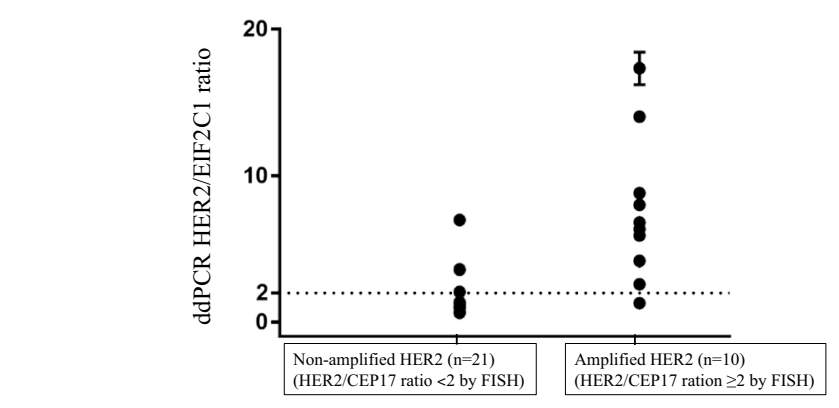

= mean value for individual sample

Fig. 2 Reproducibility of HER2/EIF2C1 ddPCR running in triplicate for individual sample from HER2-negative (non-amplified) and HER2-positive (amplified) breast cancer tissues detected by FISH. Mean \pm SD for HER2/EIF2C1 ratio is shown for individual sample. The error bar is shorter than the mean symbol in most of the samples

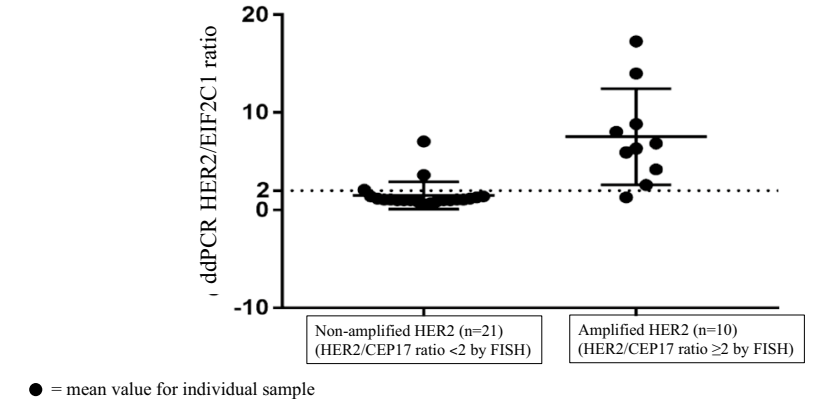

Fig. 3 HER2/EIF2C1 ratio was assessed by ddPCR on DNA from HER2-negative (non-amplified) and HER2-positive (amplified) breast cancer tissues detected by FISH. The mean values of HER2/EIF2C1 ratio for individual sample and the mean \pm SD error bar for the groups are shown 
Table 3 HER2 status in breast cancers tissues assessed by ddPCR and FISH $(n=31)$

\begin{tabular}{llll}
\hline & & \multicolumn{2}{l}{ HER2/CEP17 (FISH) } \\
\cline { 3 - 4 } & & Negative & Positive \\
\hline $\begin{array}{l}\text { HER2/EIF2C1 } \\
\text { (ddPCR) }\end{array}$ & Negative & $18(85.7 \%)$ & $1(10 \%)$ \\
& Positive & $3(14.3 \%)$ & $9(90 \%)$
\end{tabular}

The sensitivity and specificity of ddPCR assay; 90\% (9/10) and $85.7 \%$ (18/21), respectively

Acknowledgements The authors thank Professor Amnuay Thithapandha for assistance in editing the paper. Financial support of this work was by a research grant from the Ramathibodi Foundation, Ramathibodi Hospital, Mahidol University.

\section{Compliance with ethical standards}

Conflict of interest The authors declare no conflict of interests regarding the publication of this paper.

Ethical approval This study was approved by the Ethics Committee on Research involving Human Subjects of the Faculty of Medicine, Ramathibodi Hospital, Mahidol University.

Informed consent As this retrospective study was observational and link anonymized, no patient informed consent was required.

Open Access This article is distributed under the terms of the Creative Commons Attribution 4.0 International License (http://creativeco mmons.org/licenses/by/4.0/), which permits unrestricted use, distribution, and reproduction in any medium, provided you give appropriate credit to the original author(s) and the source, provide a link to the Creative Commons license, and indicate if changes were made.

\section{References}

1. Cancer Genome Atlas Network. Comprehensive molecular portraits of human breast tumours. Nature. 2012;490(7418):61-70.

2. Slamon DJ, Godolphin W, Jones LA, Holt JA, Wong SG, Keith DE, et al. Studies of the HER-2/neu proto-oncogene in human breast and ovarian cancer. Science. 1989;244(4905):707-12.

3. Revillion F, Bonneterre J, Peyrat JP. ERBB2 oncogene in human breast cancer and its clinical significance. Eur J Cancer. 1998;34(6):791-808.

4. Slamon DJ, Leyland-Jones B, Shak S, Fuchs H, Paton V, Bajamonde $\mathrm{A}$, et al. Use of chemotherapy plus a monoclonal antibody against HER 2 for metastatic breast cancer that overexpresses HER2. N Engl J Med. 2001;344(11):783-92.

5. Piccart-Gebhart MJ, Procter M, Leyland-Jones B, Goldhirsch A, Untch M, Smith I, et al. Trastuzumab after adjuvant chemotherapy in HER2-positive breast cancer. N Engl J Med. 2005;353(16):1659-72.

6. Romond EH, Perez EA, Bryant J, Suman VJ, Geyer CE Jr, Davidson NE, et al. Trastuzumab plus adjuvant chemotherapy for operable HER2-positive breast cancer. N Engl J Med. 2005;353(16):1673-84.
7. Slamon D, Eiermann W, Robert N, Pienkowski T, Martin M, Press $\mathrm{M}$, et al. Adjuvant trastuzumab in HER2-positive breast cancer. $\mathrm{N}$ Engl J Med. 2011;365(14):1273-83.

8. Swain SM, Baselga J, Kim SB, Ro J, Semiglazov V, Campone M, et al. Pertuzumab, trastuzumab, and docetaxel in HER2-positive metastatic breast cancer. N Engl J Med. 2015;372(8):724-34.

9. Verma S, Miles D, Gianni L, Krop IE, Welslau M, Baselga J, et al. Trastuzumab emtansine for HER2-positive advanced breast cancer. N Engl J Med. 2012;367(19):1783-91.

10. Wolff AC, Hammond ME, Schwartz JN, Hagerty KL, Allred DC, Cote RJ, et al. American Society of Clinical Oncology/College of American Pathologists guideline recommendations for human epidermal growth factor receptor 2 testing in breast cancer. J Clin Oncol. 2007;25(1):118-45.

11. Wolff AC, Hammond ME, Hicks DG, Dowsett M, McShane LM, Allison $\mathrm{KH}$, et al. Recommendations for human epidermal growth factor receptor 2 testing in breast cancer: American Society of Clinical Oncology/College of American Pathologists clinical practice guideline update. J Clin Oncol. 2013;31(31):3997-4013.

12. Hindson BJ, Ness KD, Masquelier DA, Belgrader P, Heredia NJ, Makarewicz AJ, et al. High-throughput droplet digital PCR system for absolute quantitation of DNA copy number. Anal Chem. 2011;83(22):8604-10.

13. Otsuji K, Sasaki T, Tanaka A, Kunita A, Ikemura M, Matsusaka $\mathrm{K}$, et al. Use of droplet digital PCR for quantitative and automatic analysis of the HER2 status in breast cancer patients. Breast Cancer Res Treat. 2017;162(1):11-8.

14. Garcia-Murillas I, Lambros M, Turner NC. Determination of HER2 amplification status on tumour DNA by digital PCR. PLoS ONE. 2013;8(12):e83409.

15. Belgrader P, Tanner SC, Regan JF, Koehler R, Hindson BJ, Brown AS. Droplet digital PCR measurement of HER2 copy number alteration in formalin-fixed paraffin-embedded breast carcinoma tissue. Clinical chemistry. 2013;59(6):991-4.

16. Zhu Y, Lu D, Lira ME, Xu Q, Du Y, Xiong J, et al. Droplet digital polymerase chain reaction detection of HER2 amplification in formalin fixed paraffin embedded breast and gastric carcinoma samples. Exp Mol Pathol. 2016;100(2):287-93.

17. Wang Y, Tsang JYS, Cui Y, Cui J, Lin Y, Zhao S, et al. Robust and accurate digital measurement for HER2 amplification in HER2 equivocal breast cancer diagnosis. Sci Rep. 2017;7(1):6752.

18. Heredia NJ, Belgrader P, Wang S, Koehler R, Regan J, Cosman AM, et al. Droplet digital PCR quantitation of HER 2 expression in FFPE breast cancer samples. Methods. 2013;59(1):20-3.

19. Tse CH, Hwang HC, Goldstein LC, Kandalaft PL, Wiley JC, Kussick SJ, et al. Determining true HER2 gene status in breast cancers with polysomy by using alternative chromosome 17 reference genes: implications for anti-HER2 targeted therapy. J Clin Oncol. 2011;29(31):4168-74.

20. Panvichian R, Tantiwetrueangdet A, Wongwaisayawan S, Nampoon A, Lertsithichai P, Leelaudomlipi S. HER2 expression in breast cancer with nonamplified HER2 and gains of chromosome 17 centromere. Appl Immunohistochem Mol Morphol. 2012;20(4):367-74.

21. Yeh IT, Martin MA, Robetorye RS, Bolla AR, McCaskill C, Shah $\mathrm{RK}$, et al. Clinical validation of an array CGH test for HER2 status in breast cancer reveals that polysomy 17 is a rare event. Mod Pathol. 2009;22(9):1169-75.

22. Marchio C, Lambros MB, Gugliotta P, Di Cantogno LV, Botta $\mathrm{C}$, Pasini B, et al. Does chromosome 17 centromere copy number predict polysomy in breast cancer? A fluorescence in situ hybridization and microarray-based CGH analysis. J Pathol. 2009;219(1):16-24.

23. Vranic S, Teruya B, Repertinger S, Ulmer P, Hagenkord J, Gatalica Z. Assessment of HER2 gene status in breast carcinomas with polysomy of chromosome 17. Cancer. 2011;117(1):48-53. 